\title{
Diagnostic Yield of Screening for Inborn Errors of Metabolism among Children Presenting with Neurologic Symptoms using Urine Amino and Organic Acid Analyses
}

\author{
Mary Anne D. Chiong, Esphie Grace D. Fodra, Judy S. Manliguis and Vanessa O. Lanot
}

Biochemical Genetics Laboratory, Institute of Human Genetics, National Institutes of Health, University of the Philippines Manila

\begin{abstract}
Objective. To review the results of urine amino and organic acid screening performed in our laboratory among patients who were referred for mental retardation, developmental delay and seizures.

Methods. A retrospective review of the results of urine amino acids and organic acids screening among patients with mental retardation, developmental delay, seizures and epilepsy was done from 2001 to 2011. Data were retrieved from the Biochemical Genetics Laboratory database.

Results. Out of 2875 children who underwent urine metabolic and urine organic acid screening from year 2001 to 2011, only $1294(45.01 \%)$ patients had clinical presentations that were related to the neurologic symptoms mentioned above. 759 (58.65\%) were boys while $535(41.35 \%)$ were girls with age range from 0-18 years old with a mean age of 2 years old. Out of this 1294 patients, 10 had Maple Syrup Urine Disease (MSUD), 3 were confirmed to have Methylmalonic Aciduria (MMA), 2 had Glutaric Aciduria type 1 (GA1), 1 had Holocarboxylase synthase deficiency, and one had Argininosuccinate Lyase (ASL) Deficiency (I). The overall diagnostic yield was $1.31 \%$.

Conclusion. Although this low yield is in concordance with what has been reported in the literature, the possibility of genetic counseling and more importantly the provision of specific treatments for those confirmed to have either an amino acid or organic acid disorder make this routine screening still highly valuable.
\end{abstract}

Key Words: inborn errors of metabolism, urine metabolic screening, urine organic acid analysis, high voltage electrophoresis, gas chromatography/mass spectrometry, mental retardation, seizures, developmental delay

\section{Introduction}

The Biochemical Genetics laboratory at the Institute of Human Genetics, National Institutes of Health is the only laboratory in the Philippines that renders services for the diagnosis and clinical management of patients with inborn errors of metabolism (IEM). This is a specialized laboratory

Corresponding author: Mary Anne D. Chiong, MD

Section of Clinical Genetics

Department of Pediatrics

Philippine General Hospital

University of the Philippines Manila

Taft Avenue, Ermita, Manila 1000 Philippines

Telephone: +6325240892

Email: maryannechiong@gmail.com that provides support and key investigations for various rare genetic disorders such as amino acid disorders, organic acid disorders, fatty acid oxidation disorders, mitochondrial disorders, peroxisomal disorders and lysosomal storage disorders. Locally available tests include urine amino acid screen by High Voltage Electrophoresis (HVE) and urine organic acid screen by Gas Chromatography/Mass Spectrometry (GC/MS). Our laboratory has also established collaboration with laboratories overseas that specialize in the testing of other IEM like fatty acid oxidation disorders, mitochondrial disorders, peroxisomal disorders and lysosomal disorders.

IEM are genetic disorders resulting from the deficiency of an enzyme, co-factor or transport proteins involved in different biochemical pathways. The biochemical defect results in an increase and decrease of important substrates causing disruption in normal bodily functions. Accumulation of the substrate of a mutant enzyme is an important cause of disease in many IEM, particularly those involving strictly degradative processes. In some disorders, the primary cause is accumulation of a normally minor metabolite, produced in excess by a reaction that is usually of trivial metabolic importance (i.e. galactitol in galactosemia). Deficiency of the product of a specific reaction is another primary consequence of inherited metabolic diseases. The extent to which it contributes to disease depends on the importance of the product (i.e. ornithine in lysinuric protein intolerance). ${ }^{1}$

Neurologic symptoms are very frequent in inborn errors and encompass progressive psychomotor retardation, seizures and number of neurologic abnormalities, in both central and peripheral system, sensorineural defects and psychiatric symptoms. A large number though present with an early and non-specific progressive developmental delay, poor feeding, hypotonia, some degree of ataxia and frequent autistic features. ${ }^{2}$ Neurologic symptoms such as psychomotor/mental retardation, developmental delay and seizures are the most frequent clinical indications for urine metabolic testing referral to our laboratory for the past 12 years.

Urine metabolic screening (UMS), composed of amino acids and organic acids analyses, is the most frequent metabolic test requested by physicians in screening for IEM 
among patients presenting with neurologic symptoms in the local setting. The most convenient but highly satisfactory preliminary investigation that may lead directly to diagnosis is to examine urinary amino acids by high voltage electrophoresis. This line of investigation provides a visible profile of amino acids and some organic acids, hence, the level of each amino acid can be analyzed semi-quantitatively and determine whether the profile exhibits a normal pattern or not. Each patient is compared to a negative control run concurrently. This test is a semi-quantitative screening mainly for amino acid disorders such as Maple Syrup Urine Disease (MSUD), Phenylketonuria (PKU), Homocystinuria, Cystinuria, Non ketotic hyperglycinemia $(\mathrm{NKH})$, among others. Organic acid analysis by Gas Chromatography/Mass Spectrometry (GC/MS) is a well-established procedure for the diagnosis of classical organic acidurias and some fatty acid oxidation defects. ${ }^{3}$

This paper presents a 10-year review of the results of urine amino and organic screens performed in our laboratory among patients who were referred for mental retardation, developmental delay and seizures.

\section{Methods}

A retrospective review of the results of urine amino acids and organic acids screening was done from 2001 to 2011. Data were retrieved from the Biochemical Genetics Laboratory database. Those patients with clinical presentation of mental retardation, developmental delay, seizures and epilepsy, were identified and the results of their urine metabolic screening and/or urine organic acid screening were reviewed.

\section{Results}

Out of 2875 children who underwent urine metabolic and urine organic acid screening from year 2001 to 2011, only $1294(45.01 \%)$ patients had findings or clinical presentations that were related to the neurologic symptoms mentioned above. 759 (58.65\%) were boys while 535 (41.35\%) were girls with age range from 0-18 years old with a mean age of 2 years old. Out of this 1294 patients, 10 had MSUD, 3 were confirmed to have Methylmalonic Aciduria (MMA), 2 had Glutaric Aciduria type 1 (GA1), 1 had Holocarboxylase synthase deficiency, and one had Argininosuccinate Lyase (ASL) Deficiency (Figure 1). Majority of the findings among patients who presented with neurologic symptoms revealed no specific amino or organic acidopathy while some presented only with non specific findings such as lactic acidosis, ketosis or both lactic acidosis and ketosis. The patients who presented with MSUD primarily had seizures, global developmental delay, epilepsy, and hypotonia. The three patients with MMA presented with seizures, global developmental delay, and hypotonia. The patients with GA1 presented with developmental delay, seizures, movement disorder and macrocephaly. The one with ASL had epilepsy. The one affected with holocarboxylase synthase deficiency presented with seizures that were difficult to control despite of anticonvulsants.

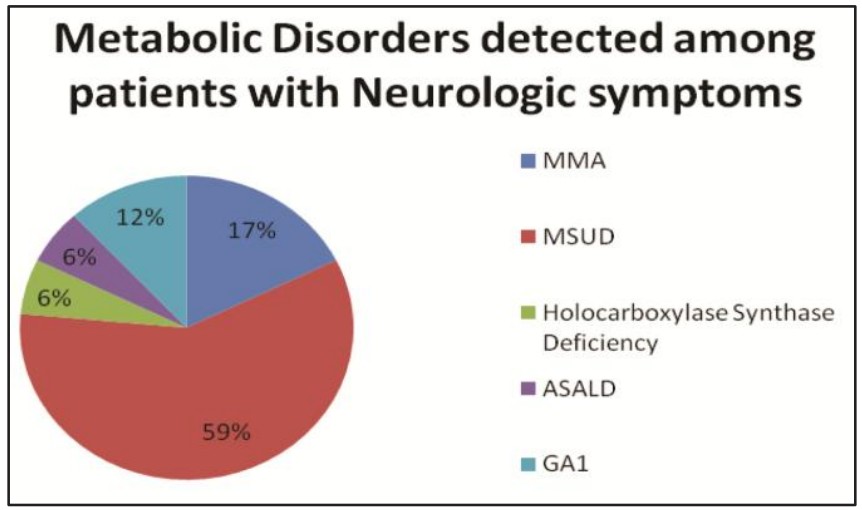

Figure 1. The metabolic disorders that were detected among patients with neurologic symptoms were MSUD, MMA, Holocarboxylase Synthase Deficiency, ASALD, and GA1. MSUD was the most common IEM detected among patients with neurologic symptoms.

The overall yield of a positive metabolic disorder among our cohort of children presenting with neurologic symptoms was $17 / 1294$ or $1.31 \%$.

\section{Discussion}

Mental retardation (MR) is a frequently occurring condition and is the third most prevalent neurologic condition after cerebral palsy and epilepsy. ${ }^{4}$ Some forms of MR have been known to be caused by biochemical disorders but establishing an etiologic diagnosis is a major challenge as the spectrum of possible causes is enormous and the range of diagnostic investigations are extensive and costly. The yield of metabolic studies for children presenting with mental retardation was low in general, with results ranging from 0.2 to $8.4 \%$ (median $1.0 \%$ ). The higher figures were from countries where specific entities are more common, such as aspartylglucosaminuria in Finland or were reached by applying checklist criteria. ${ }^{5}$ In a study determining the diagnostic yield of comprehensive assessment for patients presenting with developmental delay or mental retardation (DD/MR), the yield of routine metabolic screening of patients was likewise low at less than $1 \%{ }^{6}$

On the other hand, neurometabolic testing done on children with developmental delay in one study yielded $13.6 \%$ but findings regarding abnormal metabolites such as amino acids, organic acids as well as lactate, ammonia and pyruvate did not also lead to a diagnosis but to further investigations of metabolic studies like muscle biochemical analysis. ${ }^{7}$ Given the low diagnostic yield and high costs of metabolic testing, it is important to identify patients who will warrant testing. The key elements should include the medical, family and developmental histories, dysmorphological, ophthalmological and other neurological 
examinations, as well as appropriate use of laboratory and neuroimaging tests. ${ }^{4,5}$ It was observed that mental retardation when present with additional symptoms such as epilepsy, movement disorders, autism and other psychiatric disturbances were more likely to yield a positive result than MR alone. ${ }^{8} \quad$ Similarly, isolated epilepsy without developmental delay, mental retardation or well defined epileptic syndromes does not suggest a metabolic disorder and do not warrant metabolic investigation. ${ }^{9,10}$ Thus in our cohort patients, those who turned out positive had other symptoms apart from developmental delay, mental retardation, epilepsy or seizures alone and most of them both had mental retardation and or developmental delay with seizures.

Although our study conforms to the generally low percentage yield of metabolic screens among patients with mental retardation, developmental delay and seizures, the main factor that could have contributed to our low yield was the very limited testing that we were able to perform. In a recent web based technology on approach to treatable rare diseases with intellectual disability (ID), it was reported that $60 \%$ of IEM with ID can be detected through a panel of widely available screening tests on blood and urine (ie. aminoacids, homocysteine, copper, ceruloplasmin, organic acids, purines and pyrimidines, creatine and guanidinoacetate, glycosaminoglycans and oligosaccharides), but the remaining should still be approached in a "single test per single disease basis". ${ }^{11}$ Urine amino and organic acid screens are limited to screening of amino acid and organic acid disorders. Organelle defects such as lysosomal, peroxisomal or mitochondrial disorders which can all present with neurologic symptoms are not detected by the former examinations. Likewise, other tests such as those pertaining to creatine defects or disorders of purine/pyrimidine metabolism are not available in the local setting. A recent study although yielded a low result of $0.8 \%$ showed that in a cohort of 944 patients with mental retardation, 4 out of 7 patients had adenylosuccinate lyase (ADSL) deficiency and cerebral creatine deficiency syndromes as causes for their mental deficiencies. The rest of the patients in this particular study had phenylketonuria. ${ }^{8}$ In the local setting where newborn screening for phenylketonuria is performed, our laboratory only detected one patient with PKU using urine amino and organic screening, however, the clinical indication given for testing was cyanosis and fever. This latter patient did not undergo newborn screening.

MSUD is the most common IEM detected in our country, thus the finding that it was the most frequent IEM detected by our urine amino and organic analyses among children with neurologic symptoms was to be expected. It is an autosomal recessive disorder caused by a defect in any of the subunits of the mitochondrial branched chain $\alpha$ ketoacid dehydrogenase (BCKAD) enzyme. The metabolic block results in the accumulation of branched chain amino acids (leucine, isoleucine and valine) and their ketoacids.

Affected neonates appear normal at birth but towards the end of the first week of life present with non-specific signs and symptoms such as vomiting, lethargy, irritability and poor sucking. Without treatment, there is progressive neurological deterioration leading to death. A total of 47 local cases were previously described in the literature from 1992-2004. Majority of cases were the classic forms with onset in the first 2 weeks of life and the developmental outcome showed neurologic and developmental delays in most. ${ }^{12}$

Even though the yield of routine metabolic screen was low among our patients with neurologic symptoms, the possibility of genetic counseling and more importantly the provision of specific treatments for those confirmed to have either an amino acid or organic acid disorder make this routine screening still highly valuable.

It is recommended that after a thorough appraisal (comprehensive history including medical, family and developmental histories, physical examination, dysmorphologic and neurologic examinations and appropriate use of laboratory and neuroimaging studies) of children presenting with mental retardation, global developmental delay, seizures and the like, a standardized metabolic screening be considered as well as specific metabolic analyses in order to achieve an early diagnosis and initiate the necessary therapy.

\section{References}

1. Clarke JTR. A clinical guide to inherited metabolic diseases, 3rd ed. Cambridge University Press; 2005. pp 28-32.

2. Saudubray JM, Desguerre I, Sedel F, Charpentier C. A clinical approach to inherited metabolic diseases. In: Fernandes J, Saudubray JM, van den Berghe G, Walter J, eds. Inborn Metabolic Diseases, $4^{\text {th }}$ ed. Springer; 2006. p26.

3. Biochemical Genetics Laboratory brochure. Institute of Human Genetics-NIH Philippines.

4. García-Cazorla A, Wolf NI, Serrano M, et al. Mental retardation and inborn errors of metabolism. J Inherit Metab Dis. 2009; 32(5):597-608.

5. van Karnebeek CD, Jansweijer MC, Leenders AG, Offringa M, Hennekam RC. Diagnostic investigations in individuals with mental retardation: a systematic literature review of their usefulness. Eur J Hum Genet. 2005; 13(1):6-25.

6. Shevell M, Ashwal S, Donley D, et al. Practice parameter: evaluation of the child with global developmental delay: report of the Quality Standards Subcommittee of the American Academy of Neurology and The Practice Committee of the Child Neurology Society. Neurology. 2003; 60(3):367-80.

7. Papavasilou AS, Bazigou H, Paraskevoulakos E, Kotsalis C. Neurometabolic testing in developmental delay. J Child Neurol. 2000; 15(9):620-2.

8. Sempere A, Arias A, Farré G, et al. Study of inborn errors of metabolism in urine from patients with unexplained mental retardation. J Inherit Metab Dis. 2010;33(1):1-7.

9. Sedel F, Gourfinkel-An I, Lyon-Caen O, Baulac M, Saudubray JM, Navarro V. Epilepsy and inborn errors of metabolism in adults: a diagnostic approach. J Inherit Metab Dis. 2007; 30(6):846-54.

10. Wolf NI, Blast T, Surtees R. Epilepsy in inborn errors of metabolism. Epileptic Disord. 2005; 7(2):67-81.

11. Van karneebeek CD, Houben RF, Lafek M, Giannasi W, Stockler S. The treatable intellectual disability APP www.treatable-id.org: A digital tool to enhance diagnosis and care for rare diseases. Orphanet J Rare Dis. 2012; 7:47.

12. Lee JY, ChiongMA, Estrada SC, Cutiongco- de la Paz, EM, Silao CL, Padilla $\mathrm{CD}$. Maple syrup urine disease (MSUD)-clinical profile of 47 Filipino patients. J Inherit Metab Dis. 2008; 31 Su ppl 2:S281-5. 